\title{
Is neutrophil elastase associated with elastic tissue in emphysema?
}

\author{
B FOX,* T B BULL, * A GUZ, E HARRIS, T D TETLEY \\ From the Departments of *Histopathology and Medicine, Charing Cross and Westminster Medical School, \\ London
}

SUMMARY To test the role of elastase in the pathogenesis of emphysema human neutrophil elastase (HNE) was localised by electron microscopy using an immunogold staining technique. Specific localisation of HNE to elastic tissue in emphysema did not occur, but non-specific binding of immunoglobulin $\mathrm{G}$ (IgG) to elastic tissue in emphysematous and normal lung tissue, which was completely blocked by the non-immune serum that was homologous to the gold labelled second antibody, was found. HNE was also present, however, in the granules of neutrophils in the same sections.

Non-specific labelling associated with elastin was probably due to binding of IgG to the high numbers of hydrophobic and charged regions known to be present in this molecule, and it is concluded that our findings do not support the existence of high concentrations of elastase in association with elastin in emphysematous lung tissue.

It is widely believed that increased elastolytic activity in the interstitium of the lung is the major cause of emphysema in $\operatorname{man}^{12}$ and that neutrophil elastase has an important role in this process. Although there is unequivocal evidence that neutrophil elastase can produce emphysema in experimental animals, ${ }^{3}$ the evidence for its role in emphysema in man has mainly been circumstantial. Most of those with the disease are smokers. There is an increase in neutrophils in the peripheral blood in smokers ${ }^{4}$ and of neutrophil elastase and neutrophils in bronchoalveolar lavage fluid in smokers both with and without emphysema. ${ }^{56}$

Although we have an indication of what is happening to elastase in the capillaries and at the surface of the lung, until recently there was no evidence of what changes were taking place within the interstitium of the lung. Damiano et $\mathrm{al}^{7}$ showed an association between increased neutrophil elastase and elastic tissue in the interstitium of emphysematous lung at the electron microscopic level that was related to the severity of the emphysema present. This seemed to us such an important observation that we undertook a similar study using immunogold localisation of human neutrophil elastase (HNE) at the electron microscopic level in normal and emphysematous human lung tissue.

Accepted for publication 14 October 1987

\section{Material and methods}

Pieces of fresh human lung tissue were taken from lobectomy specimens removed for carcinoma of the lung (three men, two women, aged between 55 and 84), bronchiectasis (one man aged 20) and for paraseptal emphysema (one man aged 30). All the patients were smokers apart from the man with bronchiectasis. The lung tissue was inflated with modified Karnovsky fixative $(2.5 \%$ glutaraldehyde, $2.0 \%$ paraformaldehyde in $0.1 \mathrm{M}$ sodium cacodylate buffer) at a pressure of $25 \mathrm{~cm}$ of fixative and fixed overnight. After fixation pieces of lung tissue $(1 \mathrm{~mm})^{3}$ were sectioned, washed in sodium cacodylate-sucrose, and secondarily fixed in $1 \%$ osmium tetroxide in $0.1 \mathrm{M}$ sodium cacodylate, followed by washing in distilled water. The tissue was block stained with $2 \%$ uranyl acetate and dehydrated to absolute alcohol. The tissue was embedded in liquid TAAB epoxy resin using propylene oxide as a link reagent and polymerised at $55^{\circ} \mathrm{C}$ for 48 hours. Polymerised blocks were cut at $1 \mu \mathrm{m}$ and stained with $1 \%$ toluidine blue in $5 \%$ borax. These sections were assessed as normal $(n=5)$ and emphysematous $(n=$ 6) using a computerised image analysis system. Selected areas were cut with a diamond knife to give a light golden interference colour, mounted on 400 mesh nickel grids, and allowed to dry overnight.

Lung tissue was also prepared for comparison using the method described by Damiano et al. ${ }^{7}$ 
Polymorphonuclear neutrophils were obtained from patients with chronic myeloid leukaemia undergoing leucophoresis, and neutrophil elastase was prepared by the method of Schmidt and Havemann. ${ }^{8}$

Antibodies to HNE were raised in 3 month old New Zealand white rabbits, which had been bled via the marginal ear vein, to obtain non-immune serum. HNE $(125 \mu \mathrm{g})$ in $0.5 \mathrm{ml}$ of $0.15 \mathrm{M}$ sodium chloride was emulsified in $0.5 \mathrm{ml}$ Freund's complete adjuvant and injected subcutaneously into four sites along the flanks of each animal. Antibody titres were boosted in each animal one month later with $125 \mu \mathrm{g}$ HNE in $0.15 \mathrm{M}$ sodium chloride emulsified in Freund's incomplete adjuvant $(1 / 1, \mathrm{v} / \mathrm{v})$, and again a month later with $75 \mu \mathrm{g}$ HNE in $0.15 \mathrm{M}$ sodium chloride. Test bleeds were made and booster doses of $20 \mu \mathrm{g}$ HNE (in $0 \cdot 15 \mathrm{M}$ sodium chloride) were administered on alternate weeks until the antibody titre, which had been high, began to fall. The animals were then bled via cardiac puncture. All serum samples were tested for the presence of antibodies to HNE using Ouchterlony immunodiffusion' and the immunoglobulin fraction of the positive and pre-immune sera which had been purified by previously described methods. ${ }^{9}$

\section{ASSESSMENT OF EMPHYSEMA}

The images of the $1 \mu \mathrm{m}$ toluidine blue sections were back-projected on to a translucent glass digitiser tablet of a computerised image analysis system at a screen magnification of $\times 300$, calibrated from a stage micrometer. A series of parallel lines, drawn on a sheet of clear acetate, were laid over the projected image. The digitiser tablet pen was used to trace the length of those lines which fell across air spaces (excluding bronchi) from epithelium to epithelium. Each of these lines was defined as a linear transect. All the linear transects within the whole section were measured.

\section{IMMUNOGOLD STAINING}

Grids were floated on distilled water for five minutes and immersed in normal goat serum diluted 1 in 5 with phosphate buffered saline (PBS), at pH 7.2, containing bovine serum albumin (BSA) and Tween $20(100 \mathrm{ml}$ PBS, $0.1 \mathrm{~g} \mathrm{BSA}, 0.05 \mathrm{ml}$ Tween $20=$ PBST) for 25 minutes. Parallel grids were immersed in $1 \%$ ovalbumin (OA) in PBST. The excess blocking agent was then drained off with filter paper, the grids immersed in rabbit antihuman neutrophil elastase at a dilution of 1 in 1000 with PBST, and then incubated at $4^{\circ} \mathrm{C}$ for 72 hours. The grids were washed in several changes of PBST for 10 minutes at room temperature and then in several changes of Tris-buffered saline (TBS, $\mathrm{pH} \mathrm{8.2)}$ containing BSA and Tween 20 in similar concentrations to PBST for 10 minutes at room temperature. Gold conjugated, goat antirabbit IgG (Janssen Life Sciences Products, Wantage, United Kingdom)

( $10 \mathrm{~nm}$ in diameter) was diluted in 1 in 10 with TBST and centrifuged at $2000 \mathrm{~g}$ for 20 minutes. The grids were immersed in the supernatant which contained immunogold for one hour at room temperature and then well washed with TBS and distilled water. Sections were contrasted with Reynold's lead citrate. The immunogold labelling method described by Damiano et al, ${ }^{7}$ in which BSA was used as the sole blocking agent of non-specific binding sites, was adhered to as much as possible.

The controls used are listed in table 1. The controls used for the method of Damiano et al were (i) neutrophils present in the alveoli and capillaries and (ii) replacement of anti-HNE by PBST. Non-immune goat serum was also used as a blocking agent instead of BSA.

\section{Results}

The lung tissue sections were classed as normal or emphysematous on the basis of mean transect lengths,

Table 1 Control conditions for examination of immunogold labelling of $H N E$ in lung tissue

Control conditions

(a) Neutrophils in alveoli, interstitium and capillaries

(b) Pre-immune rabbit serum diluted 1 in 1000 in place of rabbit anti-HNE

(c) Rabbit anti-HNE preadsorbed with excess HNE as confirmed by lack of precipitate on double immunodiffusion

(d) Rabbit anti-HNE replaced by PBST

(e) Rabbit anti-HNE replaced by rabbit anti-human factor VIII

(f) Intratracheal instillation of:

(i) $10 \mu \mathrm{g} \mathrm{HNE}$ in $0.5 \mathrm{ml}$ $0 \cdot 15 \mathrm{M}$ sodium chloride, and

(ii) $0.15 \mathrm{M}$ sodium chloride into rat lungs for four minutes, then asphyxiation with carbon dioxide

(g) Incubation of:

(i) $2.5 \mu \mathrm{g} \mathrm{HNE}$

(ii) $10 \mu \mathrm{gNE}$, and

(iii) buffer with $1 \mathrm{mg}$ human lung elastin (Elastin Products, Pacific,

Minnesota, USA) in $100 \mu 1100 \mathrm{mM}$ Trishydrochloric acid, $5 \mathrm{mM}$ $\mathrm{CaCl}_{2}, \mathrm{pH} 7.0$ for two hours at $37^{\circ} \mathrm{C}$, terminated by $1000 \mathrm{~g}$ centrifugation at $4^{\circ} \mathrm{C}$ for 15 minutes, then washed three times with buffer
Reasons for controls

Positive controls for HNE in the granules

To confirm the specificity of the antibody

To show that after adsorption of the antibody to the antigen the reaction becomes negative

To detect any non-specific binding of the second antibody

To determine whether an entirely irrelevant antibody binds to elastic tissue

To establish whether our technique could localise $\mathrm{HNE}$ in lung tissue

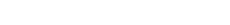

$$
\begin{aligned}
& \text { To examine binding of } \\
& \text { HNE to elastin in vitro }
\end{aligned}
$$




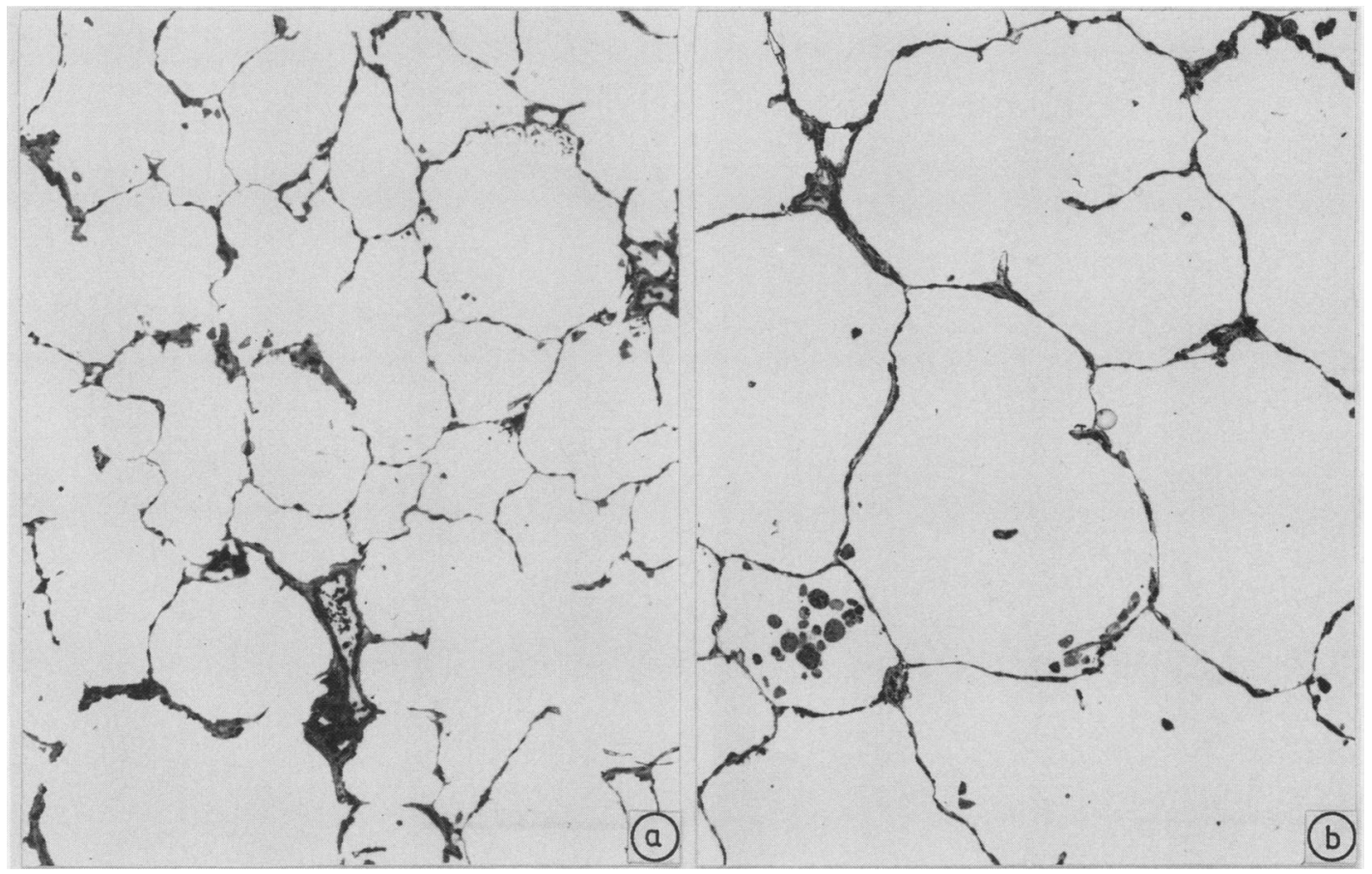

Fig 1 Human lung tissue (a) normal (b) emphysematous of sections from which electron microscopic sections were taken. (Toluidine blue).

which for normal tissue ranged from $82-108 \mu \mathrm{m}$, and for those with emphysema was 117-177 $\mu \mathrm{m}$ (Wilcoxon rank sum test, $\mathrm{p}<0.01$ ) (fig 1 ).

The results are summarised in table 2 . HNE was confined to granules of neutrophils (fig 2). There was no association between HNE and elastic tissue in normal or emphysematous lung when non-immune goat serum was used as the blocking agent (fig 3 ). In contrast, when OA/BSA was used to block nonspecific binding (table 1), both neutrophil granules and elastic tissue were labelled. The granules in the neutrophils were not labelled when pre-immune serum, PBST or antihuman factor VIII were used in place of the primary rabbit HNE. Immunogold labelled HNE was present in the lung of the rats, within neutrophils in the alveoli, and within the interstitium, but was not particularly related to elastic tissue. There was no HNE associated with elastin following incubation with elastin in vitro (fig 4). There was some residual background labelling even after blocking with goat serum mainly over nuclei, red cells, and connective tissue, but this was very slight.

When the method of Damiano et al was used there was apparent selective labelling of HNE in association with elastic tissue in normal and emphysematous lung. When non-immune goat serum was used to block nonspecific binding, however, the results were different: there was no HNE in association with elastic tissue even though it was present in neutrophil granules in the same sections. The non-specific localisation of

Table 2 Comparison of $O A / B S A$ and goat serum/BSA as blocking agents prior to immunogold labelling of $H N E$ in lung tissue

\begin{tabular}{|c|c|c|c|c|}
\hline \multirow[b]{3}{*}{ Treatment } & \multicolumn{4}{|l|}{ Blocking agent } \\
\hline & \multicolumn{2}{|l|}{$\overline{O A / B S A}$} & \multicolumn{2}{|l|}{ Goat serum/BSA } \\
\hline & Polymorphonuclear leucocytes & Elastin & Polymorphonuclear leucocytes & Elastin \\
\hline $\begin{array}{l}\text { Anti-HNE } \\
\text { Pre-immune serum } \\
\text { HNE adsorbed anti-HNE } \\
\text { Anti-factor VIII (figs 6a and b) }\end{array}$ & $\begin{array}{l}+ \\
- \\
-\end{array}$ & $\begin{array}{l}+ \\
+ \\
+ \\
+\end{array}$ & $\begin{array}{l}+ \\
- \\
-\end{array}$ & $\begin{array}{l}- \\
\overline{-} \\
-\end{array}$ \\
\hline
\end{tabular}




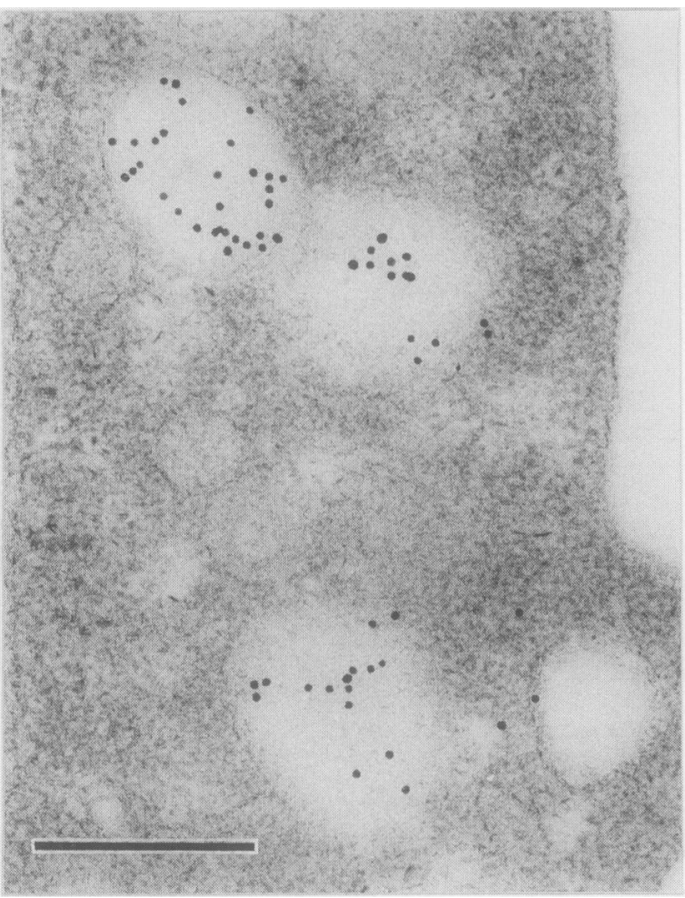

Fig 2 Immunogold labelled HNE in azurophilic granules of human neutrophil in lung capillary. Bar mark $=250 \mathrm{~nm}$.

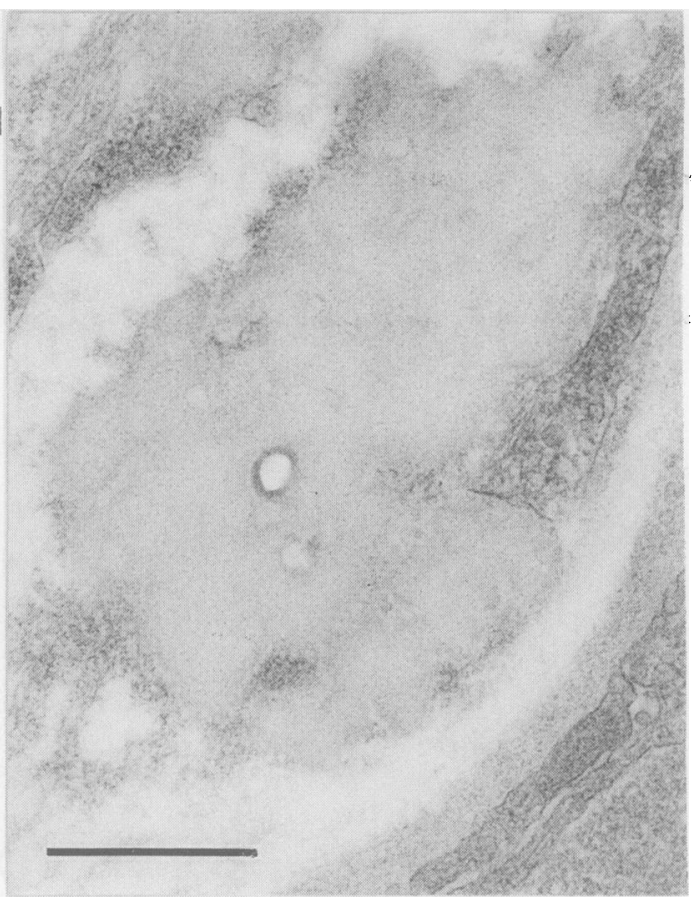

Fig 3 Elastic tissue in human lung after labelling for $H N E$ using goat serum/BSA block. No label present. Bar mark = $500 \mathrm{~nm}$.

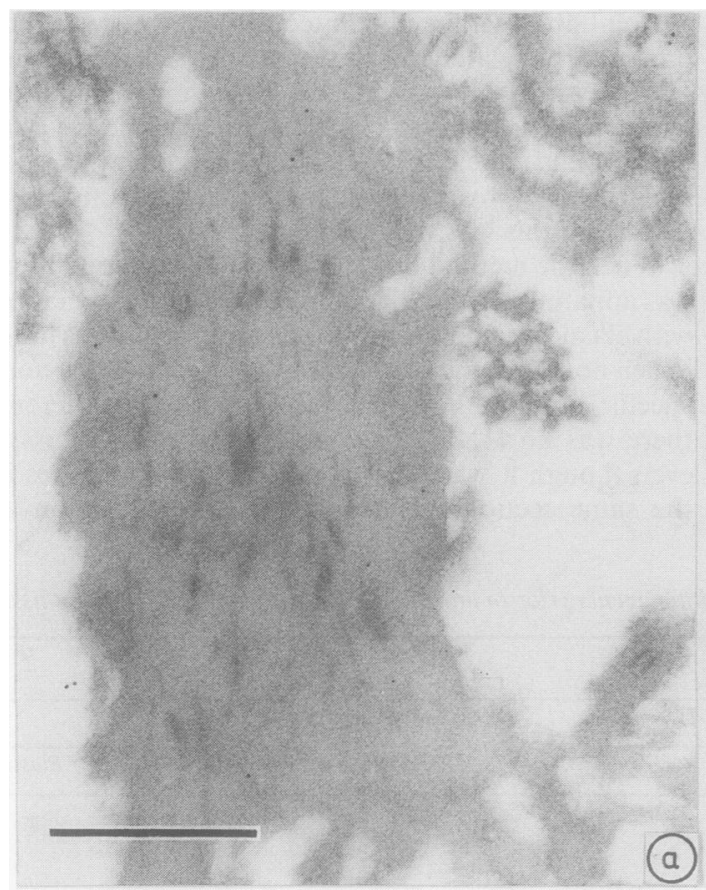

Fig 4 Human lung elastin after incubation with $H N E$ in vitro (a) using goat serum/BSA block-no label present(b) using $O A / B S A$ block-immunogold label present. Bar mark $=500 \mathrm{~nm}$. 


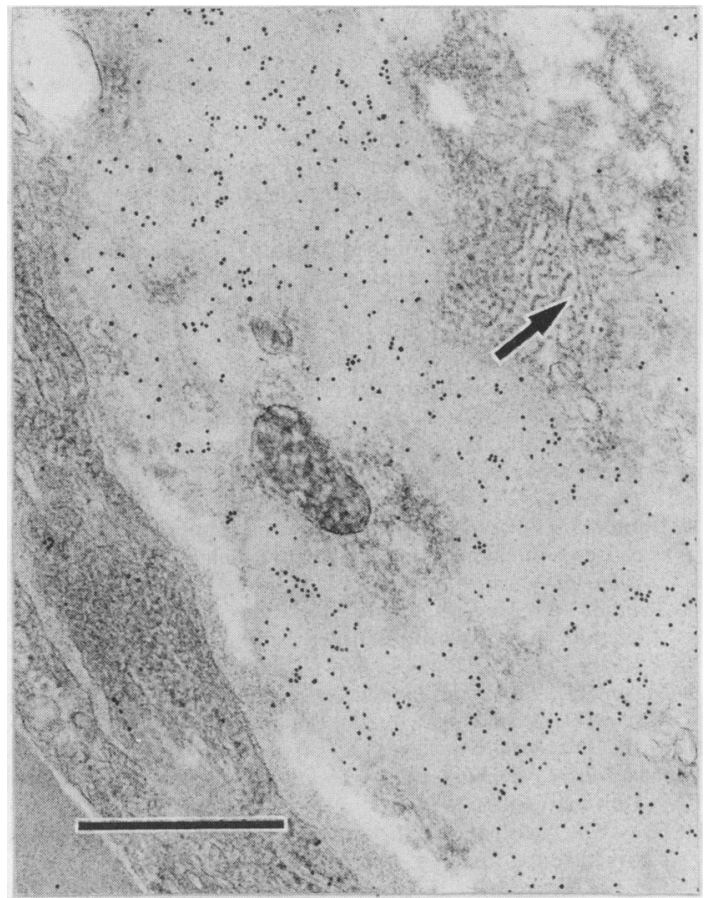

Fig 5 Human lung elastic tissue after labelling for HNE using $O A / B S A$. Label confined to amorphous elastic tissue and not microfibrillar component (arrow). Bar mark = $500 \mathrm{~nm}$. immunogold was related to the amorphous part of the elastic tissue and not the fibrillar component (fig 5).

\section{Discussion}

We were unable to confirm the findings of Damiano et al of specific localisation of HNE to elastic tissue in emphysematous human lung tissue by immunogold labelling and electron microscopy. That our method was capable of showing specific localisation of $\mathrm{HNE}$ was confirmed by the presence of HNE within granules of neutrophils in the same sections of human lung tissue, and within rat lungs after intratracheal instillation of HNE. The apparent specific localisation of HNE to elastic tissue, when OA (this study) and BSA (Damiano et $a l^{\prime}$ ) were used as blocking agents, was probably due to the non-specific binding of rabbit IgG. We have shown that this effect could be blocked by the non-immune goat sera that was homologous to the second antibody. This was confirmed when we also found that elastic tissue in the lung was capable of binding gold conjugated antirabbit serum when used without any primary antiserum.

The binding of the primary antibody to elastic tissue was probably due to the existence of hydrophobic or electrostatic forces, or a combination, ${ }^{10}$ which are normally blocked by the addition of foreign protein or the serum from the animal in which the second antibody is made - in this case the goat. BSA was incapable of completely blocking hydrophobic regions

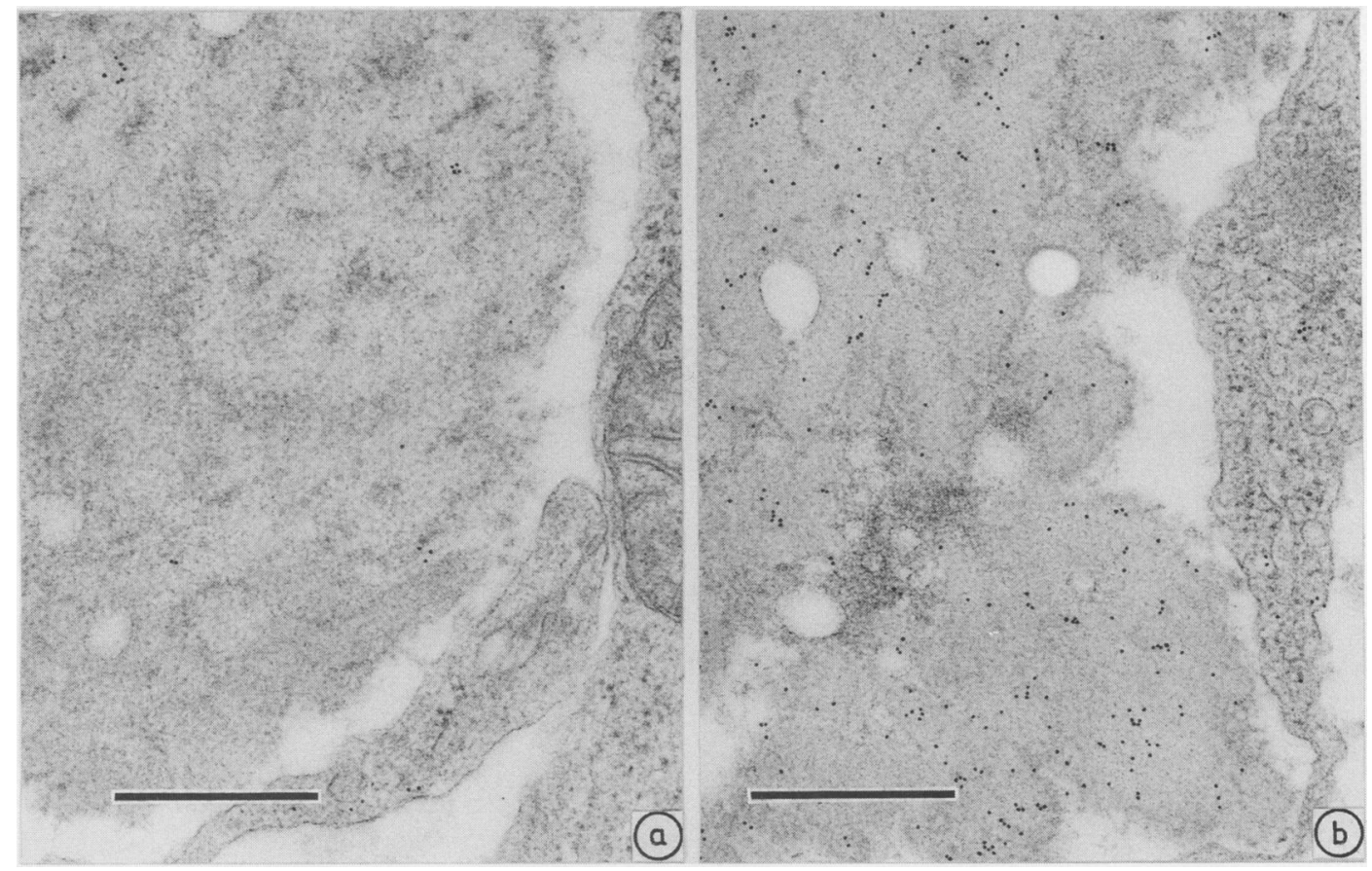

Fig 6 Human lung elastic tissue after labelling for factor VIII (a) with goat serum/BSA block showing background labelling alone (b) with OA/BSA block. Pronounced labelling of elastic tissue. Bar mark $=500 \mathrm{~nm}$. 
of the elastic molecule; these regions contain large amounts of glycine, proline, and other hydrophobic residues. ${ }^{112}$ The in vitro study described here clearly shows the inability of OA/BSA to block non-specific binding of IgG to elastin (fig 4). The fact that Damiano et al did not use goat serum as a blocking agent would account for their apparent identification of $\mathrm{HNE}$ in relation to elastic tissue.

The increased amounts of non-specific labelling found by Damiano et al with increasing severity of emphysema can also be explained thus. In emphysematous areas there may have been a change in the structure of elastic tissue with an increase in the hydrophobic regions.

Emphysema is a disease that develops slowly over many years and thus we were not surprised that we did not show residual increased concentrations of HNE associated with elastic in the advanced stages. The effect of mechanical forces and exercise for many years during breathing on elastic tissue that has already been damaged by elastase may well be responsible for the creation of the large air spaces characteristic of emphysema. That there is continued elastolysis of elastic tissue throughout life still remains to be established.

We are grateful to Dr J Moss and Mr I Shore for their advice and assistance during the study and to $\mathrm{Dr}$ M C Barrett for the preparation of the manuscript.

\section{References}

1 Janoff A. Elastases and emphysema. Am Rev Respir Dis 1985;132:417-33.

2 Tetley TD. Pulmonary emphysema. In: Malcolm ADM, ed. Molecular medicine. Oxford: IRL Press, 1984:39-62.

3 Snider GL, Lucey EC, Stone PJ. Animal models of emphysema. Am Rev Respir Dis 1986;133:149-69.

4 Sparrow D, Glynn RJ, Cohen M, Weiss ST. The relationship of the peripheral leukocyte count and cigarette smoking to pulmonary function among adult men. Chest 1984;86:383-6.

5 Janoff A, Raju L, Dearing $R$. Levels of elastase activity in bronchoalveolar lavage fluids of healthy smokers and nonsmokers. Am Rev Respir Dis 1983;127:540-4.

6 Smith SF, Guz A, Cooke NT, Burton GH, Tetley TD. Extracellular elastolytic activity in human lung lavage: a comparative study between smokers and non-smokers. Clin Sci 1985;69:17-27.

7 Damiano VV, Tsang A, Kucich U, et al. Immunolocalization of elastase in human emphysematous lungs. $J$ Clin Invest 1986;78:482-93.

8 Schmidt W, Havermann K. Isolation of elastase-like and chymotrypsin-like neutral proteases from human granulocytes. Hoppe-Seylers Z Physiol Chem 1974;335:1077-82.

9 Mayer RJ, Walker JH. Immunochemical methods in the biological sciences: enzymes and proteins. London: Academic Press, 1980.

10 Polak JM, Van Noorden S. An introduction to immunocytochemistry current techniques and problems. New York: Oxford University Press, 1984.

11 Gray WR, Sandberg LB, Foster JA. Molecular model for elastic structure and function. Nature 1973;246:461-6.

12 Foster JA, Bruenger E, Gray WR, Sandberg LB. Isolation and amino-acid sequences of tropoelastin peptides. J Biol Chem 1973;248:2876-9.

Requests for reprints to: Dr B Fox, Department of Histopathology, Charing Cross and Westminster Medical School, Fulham Palace Road, London W6 8RF, England. 